\title{
Impairment of Retention But Not Acquisition of a Visuomotor Skill Through Time-Dependent Disruption of Primary Motor Cortex
}

\author{
Arash Hadipour-Niktarash, ${ }^{1}$ Christine K. Lee, ${ }^{1}$ John E. Desmond, ${ }^{2}$ and Reza Shadmehr ${ }^{1}$ \\ Departments of ${ }^{1}$ Biomedical Engineering and ${ }^{2}$ Neurology, Johns Hopkins School of Medicine, Baltimore, Maryland 21205
}

\begin{abstract}
Learning a visuomotor skill involves a distributed network which includes the primary motor cortex (M1). Despite multiple lines of evidence supporting the role of M1 in motor learning and memory, it is unclear whether M1 plays distinct roles in different aspects of learning such as acquisition and retention. Here, we investigated the nature and chronometry of that processing through a temporally specific disruption of M1 activity using single-pulse transcranial magnetic stimulation (TMS). We applied single-pulse TMS to M1 or dorsal premotor cortex (PMd) during adaptation of rapid arm movements ( $\sim 150$ ms duration) to a visuomotor rotation. When M1 was stimulated either immediately after the end of each trial or with a $700 \mathrm{~ms}$ delay, subjects exhibited normal adaptation. However, whereas the memory of the subjects who received delayed-TMS showed normal rates of forgetting during deadaptation, the memory of those who received immediate TMS was more fragile: in the deadaptation period, they showed a faster rate of forgetting. Stimulation of PMd with adjusted (reduced) intensity to rule out the possibility of coactivation of this structure caused by the current spread from M1 stimulation did not affect adaptation or retention. The data suggest that, during the short time window after detection of movement errors, neural processing in M1 plays a crucial role in formation of motor memories. This processing in M1 may represent a slow component of motor memory which plays a significant role in retention.
\end{abstract}

Key words: TMS; motor learning; memory; motor cortex; visuomotor rotation; reaching

\section{Introduction}

Electrophysiological studies have shown that learning- and memory-related changes occur in the activity of a subpopulation of neurons in the primary motor cortex (M1) while monkeys adapt their reaching movements to force-fields or visuomotor transformations (Li et al., 2001; Paz et al., 2003). Experiments using repetitive transcranial magnetic stimulation (rTMS) have linked the early phase of motor memory consolidation to the M1 (Muellbacher et al., 2002; Baraduc et al., 2004). For example, when M1 is disrupted by $1 \mathrm{~Hz}$ rTMS for a period of $15 \mathrm{~min}$, subsequent reaching movements show normal rates of adaptation to a perturbing force-field (Richardson et al., 2006). However, the previous stimulation produces a more fragile memory, as recall of the motor skill at $24 \mathrm{~h}$ is impaired. Psychophysical data from both reaching and saccade adaptation studies suggest that there are at least two processes that contribute to motor learning and retention: a fast process that learns rapidly but has poor retention, and a slow process that learns slowly but has stronger retention (Smith et al., 2006; Kording et al., 2007). Despite these

Received June 6, 2007; revised 0ct. 23, 2007; accepted 0ct. 25, 2007.

This work was supported by National Institutes of Health Grant NS37422.

Correspondence should be addressed to Arash Hadipour-Niktarash, Department of Biomedical Engineering, Johns Hopkins School of Medicine, 410 Traylor Building, 720 Rutland Avenue, Baltimore, MD 21205. E-mail: arash.hadipour@gmail.com.

DOI:10.1523/JNEUROSCI.2570-07.2007

Copyright $\odot 2007$ Society for Neuroscience $\quad 0270-6474 / 07 / 2713413-07 \$ 15.00 / 0$ multiple lines of evidence, the chronometry of contribution of M1 to motor adaptation and memory retention remains unclear.

We hypothesized that during adaptation, disruption of activity in M1 time-locked to arrival of error information should impair the processes that learn from error. We used a single-pulse TMS protocol to briefly interfere with cortical activity in a timespecific manner relative to the event of interest during learning of a visuomotor transformation.

\section{Materials and Methods}

Subjects. Fifty-two right-handed subjects (18- to 44-years-old, $23.9 \pm 5.5$ SD, 24 females) participated after providing written informed consent. Protocols were approved by the Johns Hopkins Institutional Review Board. All volunteers were screened for history of neurological disorders and other TMS contraindications.

Apparatus and general task procedures. Subjects were seated in front of a vertically oriented monitor centered at the midline and at eye level. A sling supported the forearm in the horizontal plane, shoulder movement was restricted by a belt and the wrist was braced. Head movements were restrained with a custom-molded bite bar, and a shield prevented vision of the hand and arm.

Subjects performed rapid "shooting" reaching movements through targets displayed on the monitor while grasping the handle of a robotic arm. They were instructed to move a cursor from a central start position (depicted as a cross) to the center of the target in a straight line and with an appropriate speed. There was a 1:1 mapping between cursor and hand displacement. After a random wait time uniformly distributed between 2.5 and $3.5 \mathrm{~s}$, a beep sounded as a target $(1 \times 1 \mathrm{~cm})$ appeared. The target was located at one of three positions along a boundary circle with a $10 \mathrm{~cm}$ 
radius and the cross as the center (see below). As the cursor moved toward the target, a trace of the cursor path remained on the screen. At the moment the cursor passed through the boundary circle (trial end), the cursor was hidden, the boundary pass point (endpoint) was marked with a dot, and the robot exerted a dampening field to slow hand motion. If the cursor passed through the target, peak tangential velocity was within $130 \pm 12.5 \mathrm{~cm} / \mathrm{s}$, and path curvature index (movement path length divided by the distance between cursor position at target onset and endpoint) was $\leq 1.01$, an animation of target explosion and a tone were given as reward. The target turned red or blue if peak velocity was higher or lower than the desired range, respectively. A visual warning cue was given if the reaction time (time between target onset and movement onset) was $>400 \mathrm{~ms}$. The peak velocity feedback and the trace (up until endpoint) were displayed until the robot moved the handle back to the start position, at which point the cursor reappeared.

Experiment 1. The experiment consisted of blocks of 48 trials, with a timed period of rest (1 min) between blocks (see Fig. 1). Targets were pseudorandomly ordered within one block, so that every set of three consecutive trials included one each of the three targets at $127.5,135$, or $142.5^{\circ}$. Before the experimental session, subjects performed three blocks of familiarization in the null condition (i.e., without any visuomotor transformation). The session began with a preadaptation block in the null condition. Subjects then performed four blocks of adaptation as a rotational transformation was applied to the cursor direction with respect to the hand direction. The first 12 trials of the first adaptation block were performed with $0^{\circ}$ rotation, after which the magnitude increased by $1^{\circ}$ clockwise for every 6 trials, so that the last six trials of the fourth block were performed with the maximal $30^{\circ}$ clockwise rotation. The rotation was implemented gradually to decrease the likelihood that subjects would use cognitive strategies to improve performance (Mazzoni and Krakauer, 2006). Subjects were divided into four groups depending on the type of TMS received during the adaptation period ( $n=9$ per group). For the immediate-TMS group, we applied a single TMS pulse over M1 at the time of each trial end. For the delayed-TMS group, we applied a single pulse over M1 at $700 \mathrm{~ms}$ after trial end. Subjects in the dorsal premotor cortex (PMd) TMS group received a single pulse over PMd at the time of trial end. A fourth group of subjects did not receive TMS. After the adaptation period, all subjects performed a block of deadaptation (washout of adaptive movements back to baseline) in the null condition and in the absence of TMS.

For the delayed-TMS group, we chose the stimulation time of $700 \mathrm{~ms}$ after trial end as it was the latest time during the intertrial interval at which TMS could be reliably given without interfering with the next trial. Stimulation at later times than this tended to induce a twitch in the subject's arm that interfered with the process of placing the cursor on the cross and waiting for initiation of the next trial. For the PMd-TMS group, we sought to control for the possibility that any effects which were induced by TMS over M1 in the immediate-TMS group might be attributable to spread of the TMS-induced current from M1 to PMd. We were concerned about this because connections between the motor cortex and the premotor cortex are dense (Münchau et al., 2002), PMd plays a role in visually guided movements (Lee and van Donkelaar, 2006), and PMd cells display adaptation- and retention-related plastic changes in force field paradigms (Xiao et al., 2006). Therefore, to test this possibility, we applied TMS over PMd at an adjusted (reduced) intensity for subjects in this group (see TMS methods below).

Experiment 2. Our aim here was to consider whether a period of TMS preceding a sudden large error (e.g., initially in the deadaptation period of experiment 1) induces a greater sensitivity to the error, and whether experiment 1 was influenced by this or other nonspecific effects. As before, after familiarization training, the experiment began with a preadaptation block (48 trials/block) performed in the null condition (Fig. 1). For 8 subjects, this was followed by four blocks with a single pulse of TMS applied at the time of each trial end, but unlike experiment 1, these blocks occurred in the null condition rather than with visual rotation. In 8 other subjects, no TMS was applied. After this period, four blocks of adaptation occurred where from the first trial onwards, a $30^{\circ}$ counter-clockwise visual rotation was present. Finally, subjects performed a deadaptation block in the absence of TMS.

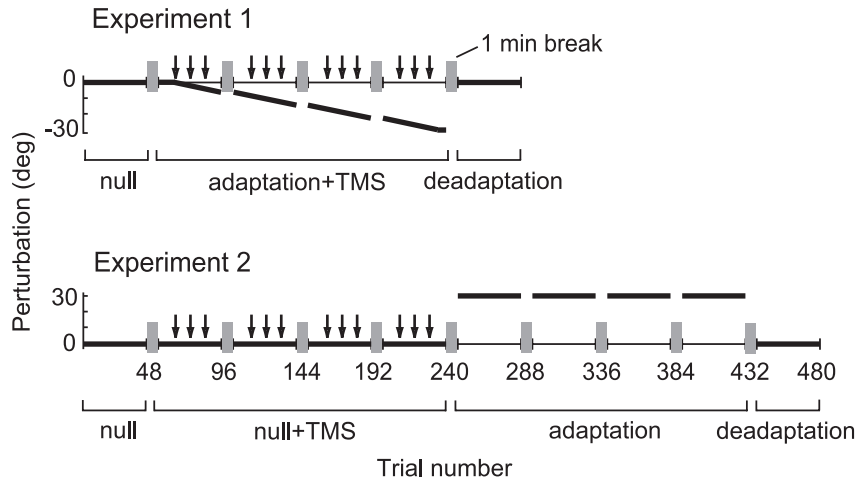

Figure 1. Experimental design. Thick lines represent visual rotation magnitude. Arrows indicate the blocks with TMS.

Targets were located at $157.5,165$, and $172.5^{\circ}$ and were pseudorandomly presented as in experiment 1 . These locations were chosen so that the initial movements of the first adaptation block had the same hand path direction as the initial movements of the deadaptation block in experiment 1 . Also, because of the counter-clockwise direction of visual rotation, the hand direction gradually shifted clockwise over the course of the block, again identical to experiment 1 . We carefully matched the movements so that, given the fixed cortical area being stimulated by TMS, the results of this experiment could not be attributed to the use of different muscles from those in experiment 1.

Analysis and statistics. We quantified performance in each trial using the angular endpoint error, defined as the angle between the line connecting the initial cursor position to the center of the target and the line connecting the initial cursor position to the endpoint. Trials were binned by three, and statistics were performed on the binned data using repeated-measures ANOVA with Tukey's correction for multiple comparisons. For calculating mean cursor trajectories, the cursor position data for each movement was resampled at 20 points evenly spaced over the movement duration (from movement onset to trial end), with linear interpolation between adjacent time points.

TMS and localization of stimulation sites. A Magstim 200 monophasic stimulator (Magstim, Whitland, UK) and a figure-of-eight coil with 70 $\mathrm{mm}$ wing diameter were used. Surface electromyogram (EMG) recordings were taken from right biceps brachii, right deltoid, and right first dorsal interosseous (FDI) muscles. The coil was placed tangentially to the scalp with the handle pointed backwards at a $45^{\circ}$ angle with respect to the anterior-posterior axis. Single pulses of TMS were applied to the left M1 to localize the FDI motor "hot spot," defined as the site that elicited maximal motor evoked potentials (MEPs) in FDI at $40-50 \%$ of maximum stimulator output. The FDI resting motor threshold (RMT) at the motor hot spot was the minimum intensity which elicited MEPs of $\geq 50$ $\mu \mathrm{V}$ amplitude in the FDI in $\geq 5$ of 10 consecutive pulses applied to the motor hot spot for the FDI muscle. The mean FDI RMT measured at the M1 hot spot for the immediate-TMS, delayed-TMS, and PMd-TMS groups in experiment 1 was $41.78 \pm 3.11,40.11 \pm 4.73$, and $41.56 \pm$ $6.89 \%$ (mean $\pm \mathrm{SD}$ ) of the maximum stimulator output respectively. Subsequently, single pulses were delivered to the left M1 to localize the biceps and the deltoid hot spots. During experiments which involved stimulation of M1, TMS was applied at $120 \%$ of FDI RMT at the midpoint between biceps and deltoid hot spots to stimulate the representation of muscles chiefly used in the present task (Thoroughman and Shadmehr, 1999). We observed TMS-induced MEPs in the right FDI, biceps, and deltoid muscles throughout the adaptation period (see Results).

For the PMd-TMS group in experiment 1, TMS was given over left PMd at an adjusted stimulation intensity to represent a coactivation of left PMd caused by current spread from TMS on left M1 (Gerschlager et al., 2001). This intensity was calculated by first finding the resting motor threshold of FDI when TMS was applied to left PMd, defined as the minimum intensity which elicited MEPs of $\geq 50 \mu \mathrm{V}$ amplitude in the right FDI muscle in $\geq 5$ of 10 consecutive pulses applied to the left PMd. The dorsal premotor cortex was localized by moving the TMS coil to a 


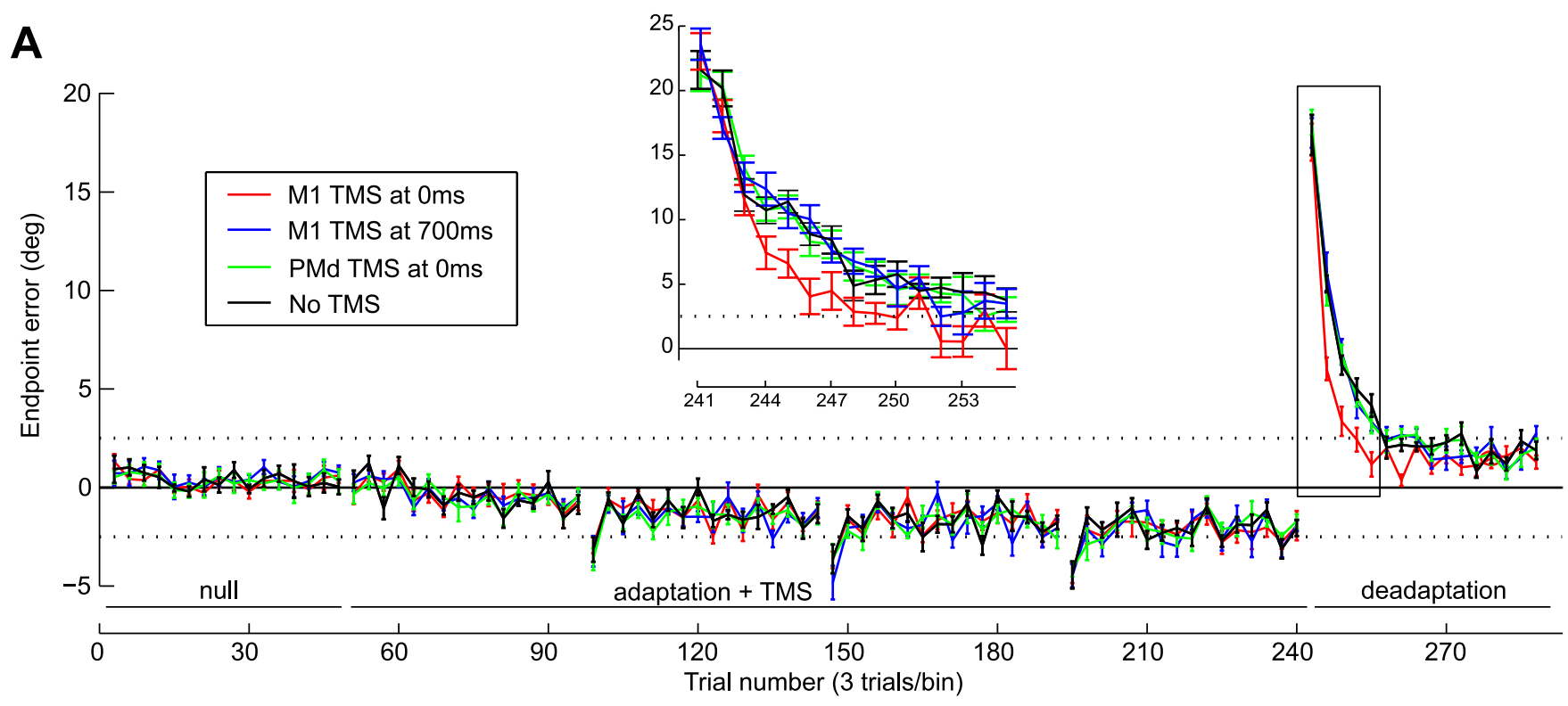

B
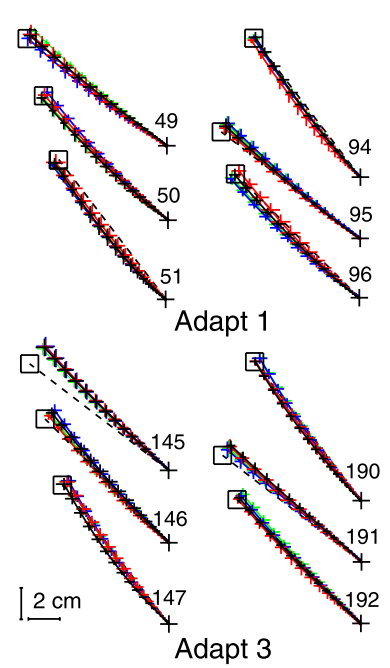
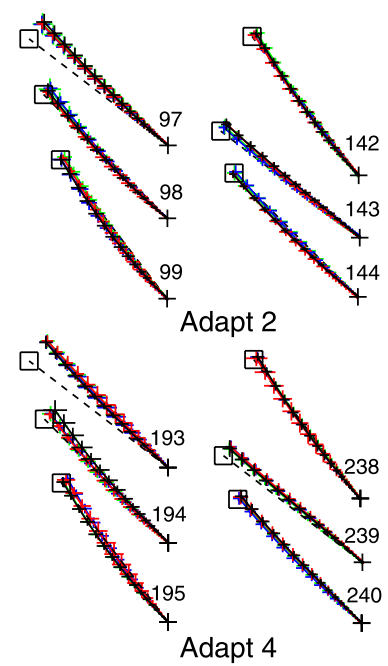

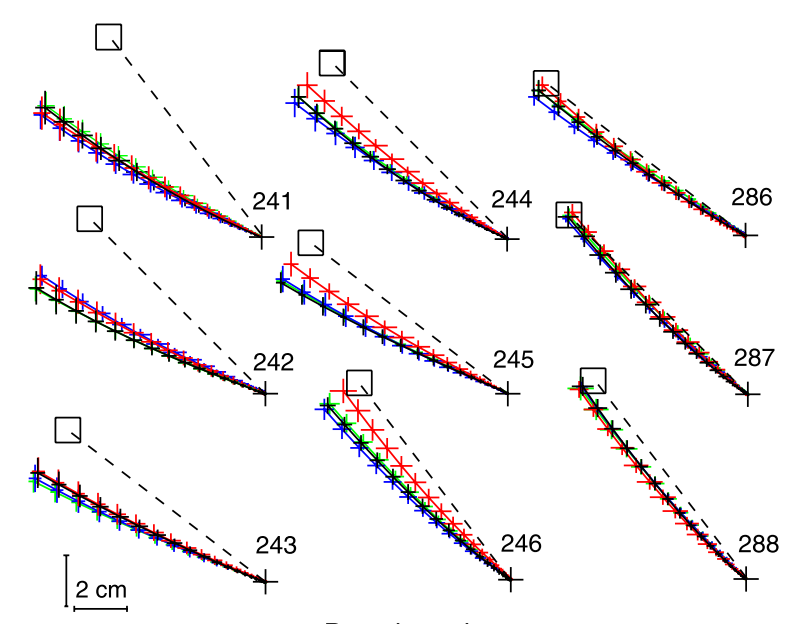

De-adaptation

Figure 2. TMS of M1 immediately after trial end impaired performance during deadaptation but not adaptation. A, Angular endpoint errors (mean \pm SEM of three-trial bins) during preadaptation, adaptation, and deadaptation periods of experiment 1. Positive values indicate counter-clockwise deviation. Inset, Expansion of box showing trial-by-trial errors (mean \pm SEM) during the early part of the deadaptation period. $\boldsymbol{B}$, Cursor trajectories (mean \pm SD) for first three and last three trials of each adaptation block, and for first six trials and last three trials of deadaptation period. Trajectory labels correspond to trial numbers.

position $2 \mathrm{~cm}$ anterior and $1 \mathrm{~cm}$ medial to the M1 hot spot for the FDI muscle. This location is based on previous functional imaging studies which have demonstrated that PMd is located $\sim 1.5-2.5 \mathrm{~cm}$ anterior to the motor cortex hand area (Fink et al., 1997; Picard and Strick, 2001). The ratio of FDI RMT measured at the M1 hot spot to FDI RMT measured at the PMd site was then found, and this ratio $(77.0 \pm 4.4 \% \mathrm{SD})$ multiplied by the value of $120 \%$ of the FDI RMT at the M1 hot spot was used as the adjusted (reduced) intensity of TMS over PMd (which corresponded to $83-98 \%$ of the FDI RMT at the hot spot).

It is possible that any observed results in the immediate-TMS group are not caused by disruption of M1 activity by TMS, but are actually the result of significant sensory sensation induced by TMS (e.g., a loud clicking sound caused by discharging of the stimulator) that nonspecifically interferes with the task (nonspecific effect) (Robertson et al., 2003). There are a number of approaches which can be used to control for such nonspecific effects. One approach is to apply TMS at different sites with the same stimulation time point. This was the case for the PMd-TMS group in experiment 1 , although it is possible that TMS over PMd induced a slightly different sensation compared with TMS over M1. Another method is to apply TMS at different time points over the same site, which was the approach taken in the case of the delayed-TMS group in experiment 1. A third approach is to use distinct tasks with stimulation at one site, which was the case for experiment 2. A final alternative is to use sham stimulation, in which the stimulator discharges with an audible clicking sound but the magnetic pulse does not traverse the skull. However, as currently available sham coils fail to produce a sensation similar to real TMS (Robertson et al., 2003), we favored the other approaches rather than a sham control.

\section{Results}

None of the subjects experienced any known side effects from TMS during the experiments. In experiment 1, we did not observe a significant effect of TMS (immediate-TMS, delayed-TMS, or PMd-TMS) on either execution or adaptation of movements (Fig. 2A). Hand trajectories appeared indistinguishable between groups for both null trials (e.g., Fig. $2 B$, trials 49-51) and visualrotation trials. Mean error of the first 12 trials of the first adaptation block was compared with mean error of the first 12 trials of the preadaptation null block to assess the effect of TMS on move- 
ment execution. We found no main effect of TMS group $\left(F_{(3,32)}\right.$ $=0.716 ; p=0.550)$ or interaction effect of group and block $\left(F_{(3,32)}=0.086 ; p=0.967\right)$. In addition, we found no significant main effects of group on movement kinematics, which included peak velocity $\left(\mathrm{PV} ; F_{(3,32)}=0.126 ; p=0.944\right)$, movement duration $\left(\mathrm{MD} ; F_{(3,32)}=0.979 ; p=0.415\right)$, path curvature index (PC; $\left.F_{(3,32)}=0.025 ; p=0.995\right)$, and reaction time $\left(\mathrm{RT} ; F_{(3,32)}=0.243\right.$; $p=0.866)$. The movement kinematics values for the entire null block were as follows: PV, immediate-TMS, $128 \pm 4.6$; delayedTMS, $129 \pm 5.6$; PMd-TMS, $127 \pm 6.2$; no-TMS, $126 \pm 6.0 \mathrm{~cm} / \mathrm{s}$, mean $\pm \mathrm{SD}$; MD, immediate-TMS, $146 \pm 16$; delayed-TMS, $156 \pm 9$; PMd-TMS, $155 \pm 11$; no-TMS, $155 \pm 11 \mathrm{~ms}$; PC, immediate-TMS, $1.0025 \pm 0.0016$; delayed-TMS, $1.0021 \pm$ 0.0014; PMd-TMS, $1.0024 \pm 0.0021$; no-TMS, $1.0025 \pm 0.0016$; RT, immediate-TMS, $297 \pm 29$; delayed-TMS, $281 \pm 39$; PMdTMS, $285 \pm 36$; no-TMS, $280 \pm 36$ ms.

Over the four adaptation blocks, errors gradually increased but remained within the target range (Fig. $2 \mathrm{~A}$, dotted lines at $\left.\pm 2.862^{\circ}\right)$. During postexperiment debriefing, we confirmed that subjects had been unaware of the gradual visual rotation, implying that the errors were attributed to self-generated variability in movements. Notably, we saw no significant effect of immediateTMS, delayed-TMS, or PMd-TMS on the extent of adaptation. First, performance of these three groups did not differ from that of the no-TMS group during the adaptation period. There was no main effect of group $\left(F_{(3,32)}=0.231 ; p=0.874\right)$ or interaction effect of group and time $\left(\mathrm{F}_{(189,2016)}=0.749 ; p=0.995\right)$ on errors of the four blocks. Similarly, kinematics remained indistinguishable between the groups (PV, $F_{(3,32)}=0.057, p=0.982 ; \mathrm{MD}$, $F_{(3,32)}=1.402, p=0.260 ; \mathrm{PC}, F_{(3,32)}=0.150, p=0.929 ; \mathrm{RT}$, $\left.F_{(3,32)}=0.579, p=0.633\right)$. Second, whereas the 1 min rest between blocks produced forgetting in all groups, the three types of TMS did not affect this time-dependent process of forgetting, as the errors of the first trials of adaptation blocks 2-4 were similar across groups (block $2, F_{(3,32)}=0.217, p=0.884$; block $3, F_{(3,32)}$ $=0.166, p=0.919$; block $\left.4, F_{(3,32)}=0.099, p=0.960\right)$. Third, when subjects performed movements during the deadaptation block after removal of the visual rotation (Fig. $2 A, B$ ), the magnitudes of the initial three aftereffects were not significantly different between groups $\left(F_{(3,32)}=0.263 ; p=0.851\right)$. Therefore, we could not detect any effects of immediate-TMS, delayed-TMS, or PMd-TMS on how the motor system adapted to the gradual perturbations.

However, in the deadaptation period, washout in the immediate-TMS group was significantly faster than the delayedTMS, PMd-TMS, and no-TMS groups, although there was no difference between these latter three groups (Fig. $2 A$ ). Here, we found a main effect of group $\left(F_{(3,32)}=8.865 ; p<0.0005\right)$ as well as an interaction effect of group and time $\left(F_{(45,480)}=2.138 ; p<\right.$ 0.0005). Tukey post hoc analysis revealed a significant difference between the immediate-TMS group and the delayed-TMS group $\left(Q_{(4,32)}=5.894 ; p=0.001\right)$, the PMd-TMS group $\left(Q_{(4,32)}=\right.$ $5.969 ; p=0.001)$, and the no-TMS group $\left(Q_{(4,32)}=6.000 ; p=\right.$ 0.001 ), whereas the other groups did not differ (delayed-TMS and no-TMS, $Q_{(4,32)}=0.031, p>0.999$; delayed-TMS and PMdTMS, $Q_{(4,32)}=0.076, p>0.999$; PMd-TMS and no-TMS, $Q_{(4,32)}$ $=0.107 ; p>0.999)$. However, we noted no significant effect of group on peak velocity PV (immediate-TMS, $125 \pm 4.5$; delayedTMS, $127 \pm 5.8$; PMd-TMS, $128 \pm 6.3$; no-TMS, $128 \pm 6.7 \mathrm{~cm} / \mathrm{s}$, mean $\left.\pm \mathrm{SD} ; F_{(3,32)}=0.611 ; p=0.613\right)$, movement duration MD (immediate-TMS, $149 \pm 18$; delayed-TMS, $160 \pm 11$; PMd-TMS, $151 \pm 8$; no-TMS, $\left.154 \pm 9 \mathrm{~ms} ; F_{(3,32)}=1.345 ; p=0.277\right)$, path curvature PC (immediate-TMS, $1.0030 \pm 0.0018$; delayed-TMS,
$1.0024 \pm 0.0014 ;$ PMd-TMS, $1.0023 \pm 0.0018$; no-TMS, $\left.1.0032 \pm 0.0020 ; F_{(3,32)}=0.511 ; p=0.678\right)$, and reaction time RT (immediate-TMS, $321 \pm 31$; delayed-TMS, $296 \pm 40$; PMdTMS, $303 \pm 40$; no-TMS, $293 \pm 42 \mathrm{~ms} ; F_{(3,32)}=0.971 ; p=$ 0.418). Therefore, although the deadaptation rate of the immediate-M1 TMS group was faster than all other groups, movement peak velocity, movement duration, path curvature, and reaction times were similar.

To examine direction-dependency of errors during deadaptation, we computed the mean error for each target direction across the trials in the first half of the block, which captured the initial rapid rate of deadaptation. A repeated-measures ANOVA with direction as within-subjects factor again found a main effect of group $\left(F_{(3,32)}=10.270 ; p<0.0005\right)$, with Tukey's post hoc analysis revealing that mean error of the immediate-TMS group was significantly different from the delayed-TMS group $\left(Q_{(4,32)}=\right.$ 6.638; $p<0.0005)$, PMd-TMS group $\left(Q_{(4,32)}=6.407 ; p<\right.$ $0.0005)$, and no-TMS group $\left(Q_{(4,32)}=6.145 ; p=0.001\right)$, whereas the other groups did not differ (delayed-TMS and no-TMS, $Q_{(4,32)}=0.493, p=0.985$; delayed-TMS and PMd-TMS, $Q_{(4,32)}$ $=0.231, p=0.998$; PMd-TMS and no-TMS, $Q_{(4,32)}=0.262, p=$ $0.998)$. There was no main effect of direction $\left(F_{(2,64)}=1.924 ; p=\right.$ $0.154)$ or interaction effect of group and direction $\left(F_{(6,64)}=\right.$ $0.245 ; p=0.960)$ on the error, implying that the effect of group was robust regardless of movement direction.

During deadaptation, at least two factors influence rates of washout: with every trial, the subject forgets some of what had previously been acquired (Smith et al., 2006) and simultaneously learns from the movement error. If the difference in the rates of washout in experiment 1 was caused by increased error sensitivity (rather than more rapid forgetting) in the immediate-TMS group, then the TMS group in experiment 2 should also learn more rapidly in the adaptation period that was preceded by TMS. However, we found that during adaptation and deadaptation (Fig. 3), there was no main effect of previous TMS. During adaptation, errors $\left(F_{(1,14)}=0.014 ; p=0.907\right)$ and movement variables $\left(\mathrm{PV}, F_{(1,14)}=0.046, p=0.834 ; \mathrm{MD}, F_{(1,14)}=2.155, p=\right.$ 0.164 ; PC, $F_{(1,14)}=0.056, p=0.817$; RT, $F_{(1,14)}=0.921, p=$ $0.354)$ did not differ with respect to the control group. There was also no interaction effect of TMS and time $\left(F_{(63,882)}=0.885 ; p=\right.$ $0.725)$ on errors during adaptation. Additionally, for the preceding null period, there was no main effect of TMS $\left(F_{(1,14)}=1.248\right.$; $p=0.283)$ or interaction effect of TMS and time $\left(F_{(63,882)}=\right.$ $0.610 ; p=0.993)$ on errors, and no main effect of TMS on movement variables $\left(\mathrm{PV}, F_{(1,14)}=0.022, p=0.883 ; \mathrm{MD}, F_{(1,14)}=\right.$ $0.134, p=0.719$; PC, $F_{(1,14)}=0.103, p=0.753$; RT, $F_{(1,14)}=$ $1.003, p=0.333)$. Finally, during the deadaptation block, there was no main effect of TMS on errors $\left(F_{(1,14)}=0.650 ; p=0.434\right)$ or movement variables $\left(\mathrm{PV}, F_{(1,14)}=0.400, p=0.537 ; \mathrm{MD}\right.$, $F_{(1,14)}=2.339, p=0.148 ; \mathrm{PC}, F_{(1,14)}=1.953, p=0.184$; RT, $\left.F_{(1,14)}=1.829, p=0.198\right)$, as well as no interaction of TMS and time on errors $\left(F_{(15,210)}=1.225 ; p=0.255\right)$.

It has been shown that in self-paced and reaction-time paradigms involving brisk thumb abduction movements, the motor cortex is more excitable during $0-160 \mathrm{~ms}$ after movement end as compared with 500-1000 ms after movement end (Chen et al., 1998). Therefore, it is possible that the difference in washout rates between immediate-TMS and delayed-TMS was because TMS disrupted M1 more effectively at trial end because of a greater excitability of M1 at that time compared with excitability after 700 ms delay. Although the present study was not explicitly designed to evaluate motor cortex excitability, a comparison of the mean amplitudes of TMS-induced MEPs in biceps brachii and 


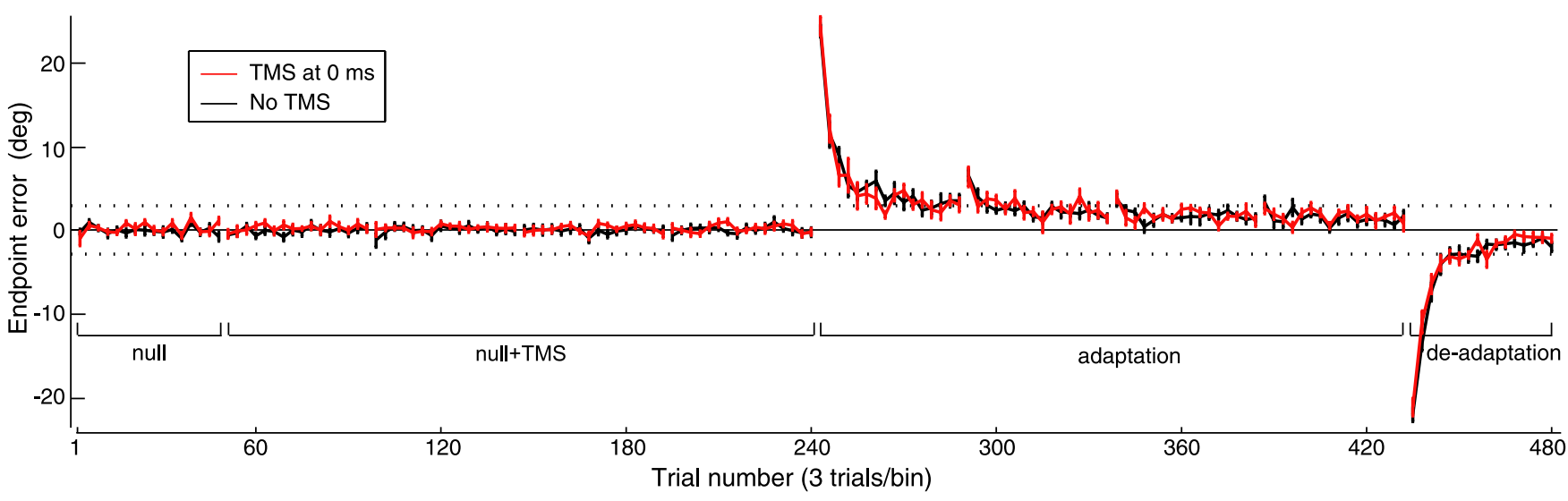

Figure 3. TMS of M1 immediately after trial end during null blocks did not affect subsequent adaptation to abrupt counter-clockwise $30^{\circ}$ visual rotation. Angular endpoint errors (mean \pm SEM of three-trial bins) during preadaptation, null with TMS, adaptation, and deadaptation periods of experiment 2 are shown.

deltoid muscles during the adaptation period of experiment 1 found no significant difference between the immediate-TMS and delayed-TMS groups for both biceps (immediate-TMS, $258.1 \pm$ $184.9 \mu \mathrm{V}$; delayed-TMS, $259.3 \pm 154.2 \mu \mathrm{V}$, mean $\pm \mathrm{SD} ; p=$ 0.796, Mann-Whitney $U$ test) and deltoid muscles (immediateTMS, $144.9 \pm 63.3 \mu \mathrm{V}$; delayed-TMS, $113.5 \pm 61.4 \mu \mathrm{V}$, mean \pm SD; $p=0.340$, Mann-Whitney $U$ test). We can infer from this that there was no significant difference between the excitability of M1 (measured through MEP amplitude) at trial end and $700 \mathrm{~ms}$ after trial end. Therefore, the faster deadaptation of the immediate-TMS group compared with the delayed-TMS group was not a result of a difference in the extent of disruption by TMS caused by greater M1 excitability. Instead, it is likely that our results were caused by temporal specificity of the disruptive effect of TMS with respect to error processing in M1.

\section{Discussion}

Previous studies have demonstrated that motor memory formation may be linked to M1 because rTMS of M1 before or after the period of adaptation can disrupt retention of a motor skill (Muellbacher et al., 2002; Baraduc et al., 2004; Richardson et al., 2006). However, because rTMS is done off-line to the adaptation period and its disruptive effect can last relatively uniformly for many minutes, rTMS experiments cannot give insight into the time when M1 contributes to memory during this period. Here we used single-pulse TMS to control for timing of disruption with respect to the event of interest (Robertson et al., 2003). We hypothesized that by disrupting M1 activity around the time of error detection, we might interfere with the process that learned from this error. However, because TMS of M1 can affect movement execution by inducing a muscle twitch, it was important to apply TMS after the movement was effectively over. Although stimulation at subthreshold intensity would prevent noticeable muscle twitch during movement, it could still affect the corticocortical and corticospinal circuit of the stimulated site (Rizzo et al., 2004), and thereby affect movement execution. We therefore required an adaptation task that, considering response latency of M1 to visual feedback (e.g., 112-192 ms) (Riehle, 1991) and to errors (e.g., 100-150 ms after onset of reaching movement with force-field perturbations) (Green et al. 2007), would allow a significant percentage of feedback to be received by $\mathrm{M} 1$ after the trial end. We designed a task involving a rapid "shooting" movement that had a task-relevant component of $\sim 150 \mathrm{~ms}$. It was found that a single-pulse of TMS over M1 at either $0 \mathrm{~ms}$ (immediately) or $700 \mathrm{~ms}$ later relative to the trial end during gradual visuomotor adaptation did not affect rates of adaptation. However, TMS at 0 $\mathrm{ms}$ resulted in a faster deadaptation rate. Remarkably, this effect on deadaptation rate was time dependent, as it did not occur when TMS was applied with a 700 ms delay.

TMS is a strong contextual cue (Robertson et al., 2003). Our main effect was found in a deadaptation period when TMS was not present. Is the more rapid deadaptation of the immediateTMS group a reflection of this change in context? Two pieces of evidence argue against this interpretation. First, TMS at $700 \mathrm{~ms}$ over M1 or at 0 ms over PMd are also strong contextual cues, but they did not affect the deadaptation rate. Second, because we applied TMS during a period of subconscious adaptation (i.e., a period when errors were kept so small as to be within the target size of the reaching movements), the change in the contextual cue (i.e., transfer from period with TMS to without TMS) was coincident with the arrival of a very large error. However, in experiment 2 we found that TMS during null blocks did not alter subsequent response to sudden large errors occurring in the absence of TMS. Therefore, it is unlikely that the faster deadaptation of the immediate-TMS group in experiment 1 was caused by a contextual change. In addition, it might be argued that immediateTMS caused a change in cortical excitability that affected the subsequent deadaptation period. Although this is already unlikely given the low frequency $(\sim 0.2 \mathrm{~Hz})$ and number of pulses (192) (Murase et al., 2005), it is ruled out by the result that the delayed-TMS group was not similarly affected by the TMS.

It is possible that the faster deadaptation rate of the immediate-TMS group was not directly related to disruption of M1 activity by TMS, but was instead caused by nonspecific effects of TMS. However, based on the results that TMS did not have an effect on performance of the delayed-TMS and PMd-TMS groups of experiment 1 or on the TMS group in experiment 2, we can conclude that the rapid deadaptation of the immediate-TMS group was specifically caused by disruption of M1 by TMS. Another possible explanation for the faster deadaptation rate of the immediate-TMS group is coactivation of PMd caused by the spread of stimulus current from TMS over M1. However, because stimulation of PMd with an adjusted (reduced) TMS intensity did not affect the deadaptation rate in the PMd-TMS group in experiment 1 , it is unlikely that coactivation of PMd contributed to the results of the immediate-TMS group.

The non-necessity of $\mathrm{M} 1$ in initial visuomotor adaptation suggested by identical adaptation rates between groups is in agreement with other studies using visuomotor and force-field para- 
digms (Paz et al., 2005; Richardson et al., 2006). However, unlike the study by Richardson et al. (2006) in which off-line TMS (before adaptation period) resulted in continuous uniform disruption of M1 activity throughout adaptation, we transiently disrupted $\mathrm{M} 1$ at two different times (0 and $700 \mathrm{~ms}$ ) relative to each trial end during adaptation. It is important to note the possibility that M1 is in fact involved in adaptation, but the disruption is being compensated by recruitment of other parts of the motor system (Lee et al., 2003).

The contribution of M1 to motor memory retention, implied by the faster deadaptation rate of the immediate-TMS group, is in some respects congruent with other studies. Muellbacher et al. (2002) and Baraduc et al. (2004) used rTMS to disrupt M1 after development of adaptation, and Richardson et al. (2006) performed rTMS to uniformly (in terms of time) disrupt M1 activity during the adaptation period. These studies found evidence for disruption of retention of the motor memory. However, unlike these studies, we disrupted M1 in a time-specific manner during adaptation. The novelty of our experiments derives from the result that the effect of TMS on motor memory retention was time dependent and no longer occurred if TMS was applied late enough in the intertrial interval (700 ms in present study). From this time-dependency, we can infer that the neural processing underlying retention of a visuomotor skill is ongoing at the end of the trial but is diminished at $700 \mathrm{~ms}$ later. This could be a potential explanation for the results of psychophysics and computational studies that have shown that the length of the intertrial interval affects motor learning (Huang and Shadmehr, 2007). Alternatively, it is possible that the processing is ongoing even 700 ms later, but is not susceptible to disruption by TMS per se. Also, because it is likely that a distributed motor network is involved in motor memory retention at different time points, it is possible that disruption of processing ongoing in $\mathrm{M} 1$ at $700 \mathrm{~ms}$ after trial end is compensated by processing occurring in other regions (Lee et al., 2003) or that processing is ongoing primarily in other regions at that time. The framework of the present study cannot dissociate between these possibilities and this issue needs more investigation.

We found that motor potentials evoked by immediate-TMS and delayed-TMS had comparable amplitudes. This suggests that there was no significant difference in M1 excitability under these two conditions and, therefore, that the faster deadaptation of the immediate-TMS group was not a result of a difference in the extent of disruption caused by greater excitability of M1. Notably, in the study by Chen et al. (1998), excitability was measured during muscle relaxation in between brisk thumb abduction movements. In the present study, the target muscles were active throughout most of the intertrial interval, including at $700 \mathrm{~ms}$ after trial end. This could explain why, unlike Chen et al. (1998), we found no significant difference in the TMS-mediated MEP amplitude between the immediate-TMS and delayed-TMS groups.

The identical adaptation rates and initial aftereffect magnitudes of control and immediate-TMS groups suggest that both groups equally acquired knowledge of the field as an internal model (Shadmehr and Mussa-Ivaldi, 1994). Considering this, what process in M1 was disrupted in the immediate-TMS group that resulted in their impaired retention? Electrophysiological experiments using visuomotor (Paz et al., 2003) and force-field (Li et al., 2001) perturbations have provided evidence for neural mechanisms in M1 that underlie retention of newly acquired skills. Disruption of these mechanisms can be considered as an explanation for the fast deadaptation rate of the immediate-TMS group. Considering the "win over" framework (Paz et al., 2003), disruption of M1 during the learning may not have affected formation of modified activities in M1 neurons, which serve to win over the default movement during adaptation. However, it may have impaired the retention of plastic changes underlying that modification, making it overwhelmed faster by default choice during deadaptation. According to the "memory cells" framework (Li et al., 2001), disruption of M1 may have impaired the proper development of plastic changes underlying class I memory cells, which normally retain the adaptive response, causing it to be balanced faster by opposite adaptive response of class II cells during the deadaptation period. In our "multiple timescale" framework (Smith et al., 2006), the fast rate of deadaptation of the immediate-TMS group may have been caused by disruption of the slow (learns slowly but with strong retention) but not the fast (learns fast but with poor retention) system, which suggests that M1 contributes to the slow processes that maintain motor memory. The presence of an intact fast system is supported by our result that forgetting occurred to a same extent across groups during rest periods in between adaptation blocks. As the slow and fast system could be reflective of class I and class II memory cells respectively (Smith et al., 2006), disruption of class I memory cell adaptive response mentioned above is congruent with disruption of the slow system.

In conclusion, the present results demonstrate that disruption of M1 during adaptation does not critically alter the adaptive response to error. However, if the disruption selectively occurs early after the end of the trial, it produces a more fragile motor memory that shows poor retention. This suggests that processing in M1 contributes to retention in a time-dependent manner, with a strong contribution early in the intertrial interval when there is a high probability of receiving error feedback, and weaker contribution at later times.

\section{References}

Baraduc P, Lang N, Rothwell JC, Wolpert DM (2004) Consolidation of dynamic motor learning is not disrupted by rTMS of primary motor cortex. Curr Biol 14:252-256.

Chen R, Yaseen Z, Cohen LG, Hallet M (1998) Time course of corticospinal excitability in reaction time and self-paced movements. Neurology 44:317-325.

Fink GR, Frackowiak RS, Pietrzyk U, Passingham RE (1997) Multiple nonprimary motor areas in the human cortex. J Neurophysiol 77:2164-2174.

Gerschlager W, Siebner HR, Rothwell JC (2001) Decreased corticospinal excitability after subthreshold $1 \mathrm{~Hz}$ rTMS over lateral premotor cortex. Neurology 57:449-455.

Green AM, Shadmehr R, Kalaska JF (2007) Motor cortex: converting errors into predications. Soc Neurosci Abstr 33:413.4.

Huang VS, Shadmehr R (2007) Evolution of motor memory during the seconds after observation of motor error. J Neurophysiol 97:3976-3985.

Kording KP, Tenenbaum JB, Shadmehr R (2007) The dynamics of memory as a consequence of optimal adaptation to a changing body. Nat Neurosci 10:779-786.

Lee JH, van Donkelaar P (2006) The human dorsal premotor cortex generates on-line error corrections during sensorimotor adaptation. J Neurosci 26:3330-3334.

Lee L, Siebner HR, Rowe JB, Rizzo V, Rothwell JC, Frackowiak RS, Friston KJ (2003) Acute remapping within the motor system induced by lowfrequency repetitive transcranial magnetic stimulation. J Neurosci 23:5308-5318

Li CS, Padoa-Schioppa C, Bizzi E (2001) Neuronal correlates of motor performance and motor learning in the primary motor cortex of monkeys adapting to an external force field. Neuron 30:593-607.

Mazzoni P, Krakauer JW (2006) An implicit plan overrides an explicit strategy during visuomotor adaptation. J Neurosci 26:3642-3645.

Muellbacher W, Ziemann U, Wissel J, Dang N, Kofler M, Facchini S, Bo- 
roojerdi B, Poewe W, Hallett M (2002) Early consolidation in human primary motor cortex. Nature 415:640-644.

Münchau A, Bloem BR, Irlbacher K, Trimble MR, Rothwell JC (2002) Functional connectivity of human premotor and motor cortex explored with repetitive transcranial magnetic stimulation. J Neurosci 22:554-561.

Murase N, Rothwell JC, Kaji R, Urushihara R, Nakamura K, Murayama N, Igasaki T, Sakata-Igasaki M, Mima T, Ikeda A, Shibasaki H (2005) Subthreshold low-frequency repetitive transcranial magnetic stimulation over the premotor cortex modulates writer's cramp. Brain 128:104-115.

Paz R, Boraud T, Natan C, Bergman H, Vaadia E (2003) Preparatory activity in motor cortex reflects learning of local visuomotor skills. Nat Neurosci 6:882-890.

Paz R, Natan C, Boraud T, Bergman H, Vaadia E (2005) Emerging patterns of neuronal responses in supplementary and primary motor areas during sensorimotor adaptation. J Neurosci 25:10941-10951.

Picard N, Strick PL (2001) Imagining the premotor area. Curr Opin Neurobiol 11:663-672.

Richardson AG, Overduin SA, Valero-Cabre A, Padoa-Schioppa C, PascualLeone A, Bizzi E, Press DZ (2006) Disruption of primary motor cortex before learning impairs memory of movement dynamics. J Neurosci $26: 12466-12470$
Riehle A (1991) Visually induced signal-locked neuronal activity changes in precentral motor areas of the monkey: hierarchical progression of signal processing. Brain Res 540:131-137.

Rizzo V, Siebner HR, Modugno N, Pesenti A, Munchau A, Gerschlager W, Webb RM, Rothwell JC (2004) Shaping the excitability of human motor cortex with premotor rTMS. J Physiol (Lond) 554:483-495.

Robertson EM, Theoret H, Pascual-leone A (2003) Studies in cognition: the problems solved and created by transcranial magnetic stimulation. J Cogn Neurosci 15:948-960.

Shadmehr R, Mussa-Ivaldi FA (1994) Adaptive representation of dynamics during learning of a motor task. J Neurosci 14:3208-3224.

Smith MA, Ghazizadeh A, Shadmehr R (2006) Interacting adaptive processes with different timescales underlie short-term motor learning. PLoS Biol 4:e179.

Thoroughman KA, Shadmehr R (1999) Electromyographic correlates of learning an internal model of reaching movements. J Neurosci 19:8573-8588.

Xiao J, Padoa-Schioppa C, Bizzi E (2006) Neuronal correlates of movement dynamics in the dorsal and ventral premotor area in the monkey. Exp Brain Res 168:106-119. 